OPEN ACCESS

Edited by:

Matteo Candidi,

Sapienza University of Rome, Italy

Reviewed by:

Michael Beyeler,

University of California,

Santa Barbara, United States

Reza Kazemi,

Atieh Clinical Neuroscience Center,

Iran

*Correspondence:

Ivy F. TSo

ivytso@umich.edu

Specialty section

This article was submitted to

Brain Imaging and Stimulation,

a section of the journal

Frontiers in Human Neuroscience

Received: 13 May 2021 Accepted: 02 August 2021

Published: 27 August 2021

Citation

Lasagna CA, Taylor SF, Lee TG, Rutherford S, Greathouse T, Gu P and Tso IF (2021) Continuous Theta Burst Stimulation to the Secondary Visual

Cortex at $80 \%$ Active Motor Threshold Does Not Impair Central Vision in Humans During a Simple

Detection Task

Front. Hum. Neurosci. 15:709275. doi: 10.3389/fnhum.2021.709275

\section{Continuous Theta Burst Stimulation to the Secondary Visual Cortex at 80\% Active Motor Threshold Does Not Impair Central Vision in Humans During a Simple Detection Task}

\author{
Carly A. Lasagna', Stephan F. Taylor', Taraz G. Lee ${ }^{2}$, Saige Rutherford ${ }^{1,3}$, \\ Tristan Greathouse ${ }^{1}$, Pan $\mathrm{Gu}^{1}$ and Ivy F. Tso ${ }^{1,2 *}$ \\ 1 Department of Psychiatry, University of Michigan, Ann Arbor, MI, United States, ${ }^{2}$ Department of Psychology, University \\ of Michigan, Ann Arbor, MI, United States, ${ }^{3}$ Department of Cognitive Neuroscience, Radboud University Medical Center, \\ Nijmegen, Netherlands
}

Continuous theta burst stimulation (cTBS) is a powerful form of repetitive transcranial magnetic stimulation capable of suppressing cortical excitability for up to 50 min. A growing number of studies have applied cTBS to the visual cortex in human subjects to investigate the neural dynamics of visual processing, but few have specifically examined its effects on central vision, which has crucial implications for safety and inference on downstream cognitive effects. The present study assessed the safety of offline, neuronavigated cTBS to V2 by examining its effects on central vision performance. In this single-blind, randomized sham-controlled, crossover study, 17 healthy adults received cTBS (at $80 \%$ active motor threshold) and sham to V2 1-2 weeks apart. Their central vision $\left(\leq 8^{\circ}\right)$ was tested at 1 - $\mathrm{min}(\mathrm{T} 1)$ and again at 50 -min (T50) poststimulation. Effects of condition (cTBS vs. sham) and time (T1 vs. T50) on accuracy and reaction time were examined using Bayes factor. Bayes factor results suggested that cTBS did not impair stimulus detection over the entire central visual field nor subfields at T1 or T50. Our results offer the first explicit evidence supporting that cTBS applied to V2 does not create blind spots in the central visual field in humans during a simple detection task. Any subtler changes to vision and downstream visual perception should be investigated in future studies.

Keywords: transcranial magnetic stimulation, theta burst stimulation, vision, safety, V2, visual cortex

\section{INTRODUCTION}

Transcranial magnetic stimulation (TMS) is a powerful, non-invasive technique for modulating cortical activity. When multiple pulses are delivered in patterned succession, as occurs in repetitive TMS (rTMS), it can excite or inhibit cortical activity within focal regions for sustained periods (Eldaief et al., 2011). In the field of cognitive neuroscience, low-frequency rTMS protocols (e.g., $1 \mathrm{~Hz}$ ) - capable of eliciting long-term inhibition in the human cortex (Klomjai et al., 2015) - have become a popular non-invasive tool for elucidating the neural mechanisms of mental processes 
through induction of "virtual lesions." In 2005, a patterned form of rTMS - continuous theta burst stimulation (cTBS), comprised of three pulses of stimulation at $50 \mathrm{~Hz}$ delivered every $200 \mathrm{~ms}$ for $40 \mathrm{~s}$, for a total of 600 pulses (Huang et al., 2005) - has gained attention because this specific pattern is capable of suppressing activity for longer periods (up to $50-\mathrm{min}$ ) with briefer administration times than more non-patterned stimulation (40 s vs. 10-20 min) (Huang and Rothwell, 2004; Huang et al., 2005; Nyffeler et al., 2006; Thut and Pascual-Leone, 2010). As such, cTBS provides a quick, non-invasive, and well-tolerated (Huang et al., 2005) means to test the causal roles of specific brain regions in functional abnormalities observed in traumatic brain injuries or neuropsychiatric disorders.

The inhibitory effects of cTBS have been well-studied in motor cortices (Huang et al., 2005). Recent evidence suggesting that cTBS similarly suppresses cortical excitability when applied to visual cortex (Franca et al., 2006; Brückner and Kammer, 2016) has prompted studies to use cTBS to advance our understanding of brain dynamics underlying visual processing. For example, applications of cTBS to late-stage visual areas such as V5 have shown modulation of higher-level (global motion) processing without impairing lower-level (local motion) perception (Cai et al., 2014). However, when administering cTBS on early visual areas (i.e., V1-V3) to examine downstream effects, one critical challenge arises. Studies on cats have shown that brief TMS pulse trains $(1-8 \mathrm{~Hz}$ for $1-4 \mathrm{~s})$ to early occipital regions can generate visual field defects or scotoma for up to 10-min (Allen et al., 2007). Given cross-species similarities in the organization of these early areas (McKeefry et al., 2009), it is possible that cTBS applied to early visual areas in humans can also impair vision for extended periods. Without confirming the integrity of vision following stimulation, altered performance on visual tasks (Bertini et al., 2010; Rahnev et al., 2013; Cai et al., 2014; Chiou and Lambon Ralph, 2016; Chen et al., 2020) after cTBS to visual cortices could be merely due to blind spots, rather than the hypothesized mechanism of reduced feedforward activity from early visual areas to later processing areas. Impaired vision not only threatens the internal validity of the findings, but also constitutes a safety issue for human subjects.

Relatively few studies applying cTBS to early visual areas in humans have examined its effects on vision. Among those that have, many have focused on more peripheral locations in the visual field. From both the safety and scientific integrity perspectives, blind spots to the central visual field would be more problematic and thus warrant more attention for investigation. Additionally, findings from these studies have been inconsistent - reporting impairments (Rahnev et al., 2013; Cai et al., 2014; Chiou and Lambon Ralph, 2016; Chen et al., 2020), improvements (Waterston and Pack, 2010; Clavagnier et al., 2013), and non-significant changes (Waterston and Pack, 2010; Brückner and Kammer, 2014; Kaderali et al., 2015; Abuleil et al., 2021) to vision. This is likely due to variability in methods, including differences in stimulation intensity, performance measurement, target localization, and task demands. In many cases, these results were also based on extremely small samples (many had $N<10$ ) (e.g., Waterston and Pack, 2010; Brückner and Kammer, 2014; Kaderali et al., 2015; Abuleil et al., 2021), and therefore negative findings could reflect a failure to reject the null hypothesis due to limited power rather than a true absence of stimulation effect on vision. As a result, it is unclear whether cTBS can be safely applied to early visual areas without inducing transient blind spots in central vision.

The present study assessed the effects of cTBS to an early visual area, V2, on central vision under a "typical" stimulation intensity level used in the majority of cTBS studies, $80 \%$ active motor threshold (Turi et al., 2021). We used a single-blind crossover design, in which participants received both conditions (randomized to receive either cTBS first or sham first) and underwent central vision testing at 1-min and 50-min poststimulation. V2 was targeted because it is one of the earliest visual processing regions and has strong feedforward connections to higher-level processing areas in both the dorsal and ventral visual streams. We tested central vision (visual angle $\leq 8^{\circ}$ ) which is critical to performing most visual perception tasks and may be particularly susceptible to cTBS effects due to the size and depth of the cortical surface it occupies (Raz and Levin, 2014). We stimulated at $80 \%$ of active motor threshold (AMT) because the majority of cTBS studies use AMT to determine stimulation intensity, and of those, $\sim 90 \%$ utilize an intensity of $80 \%$ AMT (Turi et al., 2021). Thus, cTBS to V2 at $80 \%$ AMT can be considered a "standard" intensity for V2-cTBS and will allow us to determine whether central vision changes with safety and data integrity implications (i.e., causing blind spots) occur under a typical cTBS protocol.

Lesion studies in macaques show that V2 has unique functionality in visual detection dependent on task demands V2 lesions impair complex but spare basic stimulus detection (Merigan et al., 1993). Here, "basic detection" requires accurate distinctions between coarsely discriminable stimuli (e.g., discriminate horizontal from vertical lines displayed on plain background), while "complex detection" places more demands on the visual system (e.g., judge orientation of shapes comprised of disconnected dots displayed against background distractors). Thus, we hypothesized that cTBS to V2 in humans would not impact detection accuracy on a basic visual task. Given previous reports of slowed visual detection following cTBS to early occipital targets (Fiori et al., 2015), we hypothesized that reaction time (RT) would increase after cTBS.

\section{METHODS}

\section{Participants}

Participants were $N=17$ healthy adults (six females; age $24.8 \pm 8.6$; education $16.5 \pm 2.3$ years). Inclusion criteria consisted of the following: ages $18-55$, visual acuity equal to or better than $20 / 30$ on a Snellen chart, intact central/peripheral vision (test details below), no contraindication to TMS (see Rossi et al., 2009) or MRI, not taking psychotropic medication, no medical conditions with neurological sequelae (e.g., traumatic brain injury), no prior mental illness according to Structured Clinical Interview for DSM-IV-TR, non-patient version [SCID-NP (First et al., 2002)], and no substance abuse 
in past month (according to SCID-NP; corroborated by passing urine drug screenings in each visit).

\section{Procedure}

The study protocol was approved by the Institutional Review Board at the University of Michigan Medical School and conducted in accordance with the Declaration of Helsinki. Prior to data collection, written informed consent was collected from all participants.

Participants completed three sessions: baseline, cTBS, and sham (Figure 1). Order for cTBS and sham sessions was counterbalanced across participants. At baseline, each participant completed a screening assessment, high-resolution T1-weighted (T1w) and T2-weighted (T2w) structural MRI scans, and a procedure to determine active motor threshold. Vision tests (central, acuity, peripheral) were administered to allow participants to become acclimated with the tasks and to confirm all had normal vision. TMS was delivered with a MagVenture MagPro X100 $70 \mathrm{~mm}$ figure-8 shaped TMS coil (MCF-B70). In each stimulation session (cTBS, sham), central vision was tested $\sim 1$ min after stimulation ("T1") and then again at $\sim 50$-min ("T50") after completing a $\sim 40$-min fMRI scan. Note that the fMRI scans/tasks were unrelated to the research question being addressed in this paper (whether cTBS applied to V2 at $80 \%$ AMT causes blind spots) and thus are outside the scope of the present paper and will be reported elsewhere. Beyond monitoring for common adverse events associated with TMS (e.g., headache, syncope), we also tested for additional vision-related adverse events following cTBS (and sham) that would pose a safety concern for participants. These "vision safety checks" involved a peripheral vision test and a visual acuity test that were administered at the end of each stimulation session, to ensure intact vision and safety before participants leave the laboratory.

\section{Transcranial Magnetic Stimulation Intensity}

Stimulation intensity for the cTBS or sham was based on the participant's active motor threshold (AMT), determined as the lowest intensity eliciting motor-evoked potentials of the first dorsal interosseous muscle (right hand; $20 \%$ maximum voluntary contraction) $\geq 100 \mu \mathrm{V}$ on $5 / 10$ trials. Mean raw AMT across participants was $36 \pm 6 \%$ of maximum stimulator output (MSO).

\section{Target Localization}

Individual structural brain images were used to localize V2. Highresolution T1w and T2w anatomical images $[256 \times 256 \mathrm{FOV}$, 208 slices, $1 \mathrm{~mm}$ isotropic voxels, PROMO correction (White et al., 2010)] were acquired using a GE (MR750 DV25.0) 3T scanner and used to generate subject-specific masks of V2 and V1 (to facilitate determination of V2 boundaries). Details of image processing are provided in the Supplementary Material.

As shown in Figure 2A, V1 and V2 masks (right hemisphere) were superimposed on the native $\mathrm{T} 1 \mathrm{w}$ volume in Brainsight software (Version 8; Rogue Research Inc., Montreal, QC, Canada). A target was placed in the center of a gyrus within the V2 cortex. Care was taken to avoid V1 and to ensure coil placement that minimized the scalp-to-target distance, as E-field strength reduces as a function of distance (Ilmoniemi et al., 1999). Across participants, the average distance from coil to target was $16.9 \pm 3.7 \mathrm{~mm}$. Figure 2B shows V2 targets for all subjects.

\section{Continuous Theta Burst Stimulation}

Continuous theta burst stimulation was delivered to V2 off-line at $80 \%$ of AMT with the coil oriented perpendicular to the gyrus of the target to optimize effects of the E-field (Janssen et al., 2015). Average stimulation intensity across participants was $29 \pm 5 \%$ of MSO. cTBS parameters consisted of three $50 \mathrm{~Hz}$ pulse trains delivered every $200 \mathrm{~ms}$ continuously for $40 \mathrm{~s}$, totaling 600 pulses (Huang et al., 2005). Brainsight Neuronavigation software with Polaris 3D Tracking (Version 8; Rogue Research Inc., Montreal, QC, Canada) utilizing T1w images enabled precise localization of target-centric subject/coil tracking during stimulation.

During stimulation, participants' forehead was stabilized against a headrest to minimize movements. Given that research on state-dependent effects suggest that TMS to visual areas (V1V4) maximally affects neuronal populations that are minimally active during stimulation (Silvanto et al., 2008), cTBS was administered while participants were blindfolded to control visual sensory input. For sham, the coil was rotated $90^{\circ}$ so that the handle was perpendicular to the scalp.

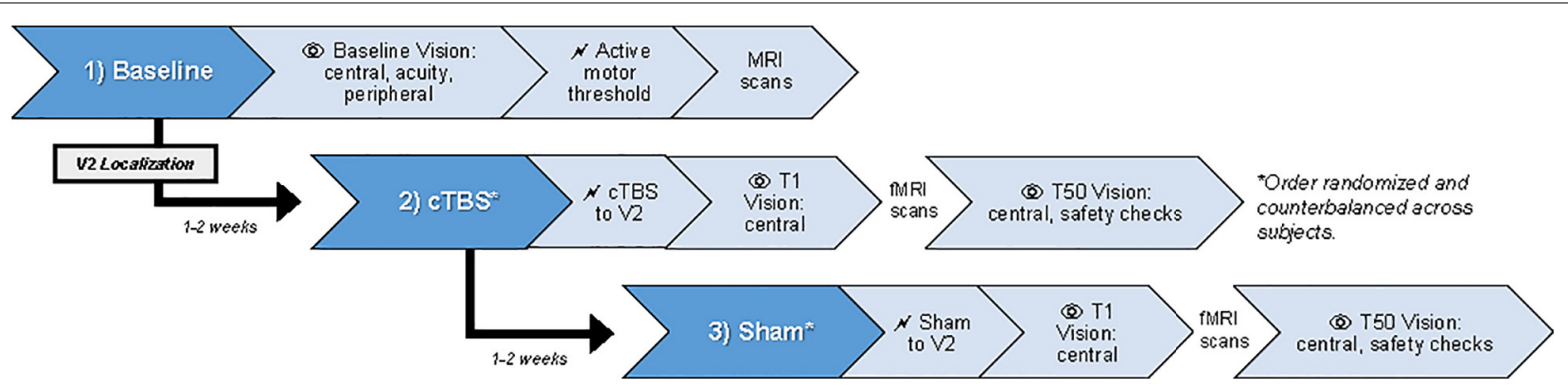

FIGURE 1 | Overview of study procedures. Participants completed three sessions: baseline, cTBS, and sham-cTBS. Session order was randomized and counterbalanced across participants. T1 and T50, 1- and 50-min post-stimulation; cTBS, continuous theta burst stimulation; V2, secondary visual cortex. MRI scans were collected on Day 1 to guide target selection and TMS neuronavigation during subsequent visits. Note that the fMRI scans/tasks were unrelated to the research question being addressed in this paper (whether cTBS applied to V2 at 80\% AMT causes blind spots) and thus are outside the scope of the present paper and will be reported elsewhere. 
A

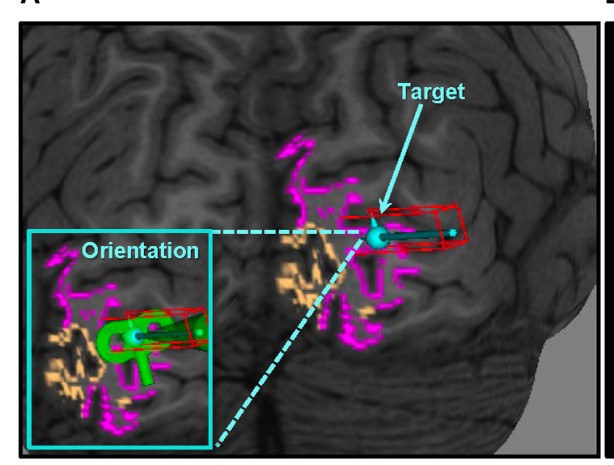

B

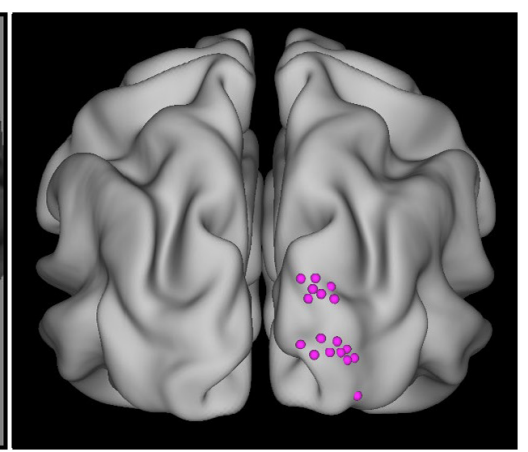

FIGURE 2 | V2 localization overview. (A) Target selection for a single representative subject. Individualized overlays were superimposed on the T1 anatomical image (native space) - V1 colored orange, V2 colored pink - and used as guides in the V2 localization process; (B) V2 targets for all participants standardized in MNI space (average location: $x=21 \pm 5, y=-95 \pm 6, z=19 \pm 12$; see Supplementary Material for individual subject coordinates). V1, primary visual cortex; V2, secondary visual cortex.

\section{Vision Tests}

\section{Central Vision Task}

A computerized task was used to examine binocular detection of visual stimuli across the central visual field $\left(\leq 8^{\circ}\right)$. Participants looked at a central fixation $\left(\right.$ size $=1^{\circ}$ ) while target stimuli (" 1 " or " 2 "; size $=0.75^{\circ}$ ) flashed briefly for $50 \mathrm{~ms}$ at different locations, one at a time, on the screen. Participants pressed the number key " 1 " or " 2 " to indicate what they saw and were given an indefinite period of time to respond. Following a response, a $200 \mathrm{~ms}$ interval elapsed before the next trial. Each trial was signaled by a $50 \mathrm{~ms}$ tone to minimize attention lapses. Modeled on Barendregt et al. (2014), targets were presented at 32 locations across the central visual field (Figure 3). These locations covered four visual angle eccentricities $\left(2^{\circ}, 4^{\circ}, 6^{\circ}\right.$, and $\left.8^{\circ}\right)$ and eight polarities rotated about a central fixation $\left(22.5^{\circ}, 67.5^{\circ}, 112.5^{\circ}, 157.5^{\circ}, 202.5^{\circ}\right.$, $247.5^{\circ}, 292.5^{\circ}$, and $337.5^{\circ}$ ). Five presentations occurred at each of the 32 locations in randomized order, resulting in 160 trials and a task duration of $\sim 3$-min. The task was presented in Psychtoolbox-3 (Brainard, 1997; Pelli, 1997; Kleiner et al., 2007) in MATLAB (R2019a) using a HP EliteBook laptop (Windows 10, $1920 \times 1080$ resolution, $33.5 \mathrm{~cm} \times 17.5 \mathrm{~cm}$ display, $60 \mathrm{~Hz}$ refresh rate, 6-bit color depth) placed at an eye-level viewing distance of $55 \mathrm{~cm}$. A headrest was used to minimize movements. Vision performance was evaluated by accuracy and RT for each stimulation condition (cTBS, sham) and administration time (T1, T50).

\section{Vision Safety Checks}

The integrity of peripheral vision was assessed monocularly using an experimenter-administered visual confrontation task (see Supplementary Material for description). Normal peripheral vision (requisite for participation) was defined as reliable identification on four trials in all quadrants of both eyes at baseline. Visual acuity was tested binocularly using a Snellen chart. Normal visual acuity (requisite for participation) was defined as 20/30 or better, with corrective lenses if needed. When re-tested after cTBS and sham stimulation sessions, any reductions in acuity or identification of stimuli in a quadrant were used to test for safety concerns related to stimulation.

\section{Statistical Analyses}

The question of whether cTBS applied to an early visual area induces (temporary) blindness can be more meaningfully addressed by evaluating the comparative evidence for the null vs. alternative hypothesis. This can be achieved only by Bayesian analysis and not traditional null hypothesis significance testing. Therefore, we used a Bayesian model comparison approach to evaluate the relative strength of evidence for the null and alternative hypotheses (Quintana and Williams, 2018). We compared central vision performance following cTBS vs. sham by calculating the Bayes Factor (BF) - the ratio of Bayesian evidence for the alternative model (cTBS-related vision changes) to the null model (no cTBS-related vision changes). $\mathrm{BF}<1$ offers evidence for the null and $\mathrm{BF}>1$ offers evidence for the alternative. The strength of evidence is interpreted as follows (Jarosz and Wiley, 2014): evidence for null $=0.33-1$ (anecdotal), 0.10-0.33 (substantial), 0.033-0.10 (strong), 0.01-0.033 (very strong), <0.01 (decisive); evidence for alternative $=1-3$ (anecdotal), 3-10 (substantial), 10-30 (strong), 30-100 (very strong), >100 (decisive).

We used the "anovaBF" function in the "BayesFactor" (Morey and Rouder, 2015) R package to compute the BF of each possible alternative model with Stimulation (cTBS, sham) and Time (T1, T50) as possible fixed factors and subjects as a random effect, against the null model (subjects as random effect only) (Morey and Rouder, 2015). This was run separately for accuracy and RT as dependent variable. All outputs are provided in Supplementary Material and $\mathrm{R}$ analysis code is available at https://osf.io/3vxyn/.

\section{RESULTS}

Continuous theta burst stimulation to V2 was well-tolerated and no participants reported common adverse side effects related to 


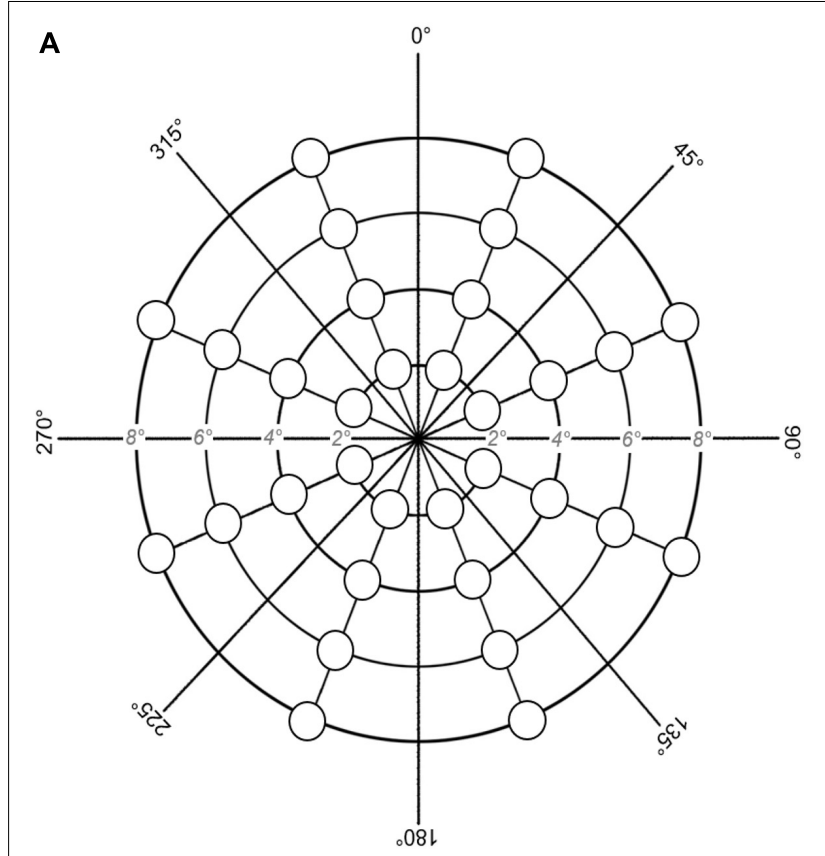

B

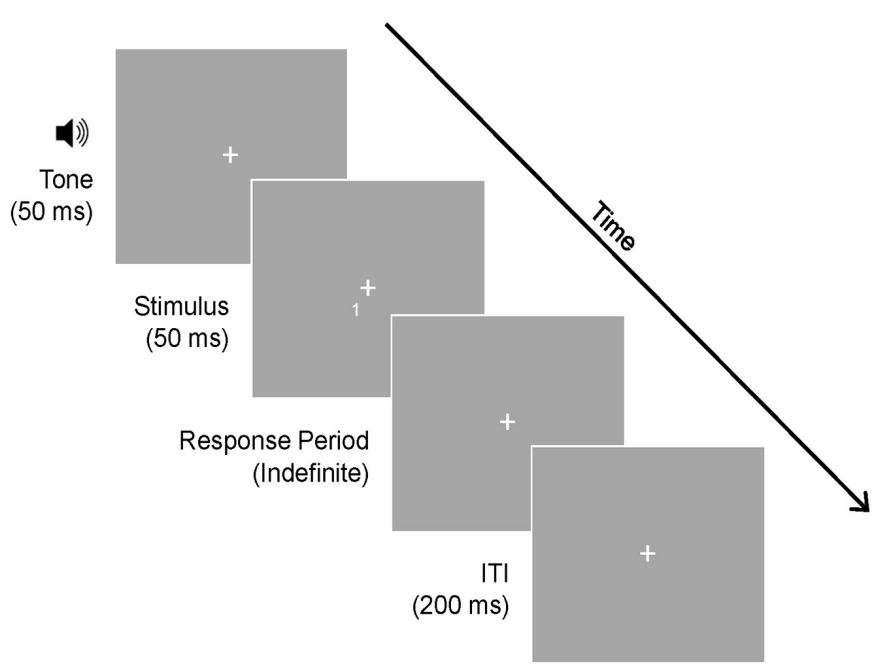

FIGURE 3 | Central vision task. (A) Stimulus presentation locations. (B) Trial structure of the task. Each stimulus was preceded by a $50 \mathrm{~ms}$ tone, presented briefly for $50 \mathrm{~ms}$, and followed by an indefinite response period. A $200 \mathrm{~ms}$ inter-trial interval (ITI) elapsed between trials. Five stimulus presentations occurred at each of the 32 locations shown, resulting in 160 trials. ms, milliseconds.

TMS (e.g., headache, syncope). Our vision safety checks revealed that no participants experienced reductions in visual acuity or impaired peripheral vision at the end of cTBS (or sham) visits. Accuracy and RT results are summarized in Figure 4.

\section{Accuracy}

Accuracy on the central vision task was high both after cTBS $(\mathrm{T} 1=98.4 \pm 5.8 \% ; \mathrm{T} 50=97.2 \pm 8.2 \%)$ and sham $(\mathrm{T} 1=98.3 \pm 5.8 \%$; T50 $=97.9 \pm 6.7 \%)$. Similarly, accuracy at all individual stimulus locations was also high (above $90 \%$ for all locations in both conditions). Vision performance for individual subjects is provided in Supplementary Material.

The null model had stronger evidence than all six models that contained Stimulation (as main effect or interaction with Time). Specifically, the evidence for the null model was "substantial" relative to three of these alternative models $(\mathrm{BF}=0.31$ to 0.14$)$ and "anecdotal" relative to the other three alternative models $(\mathrm{BF}=0.92$ to 0.61$)$. Together, the results suggest that cTBS to V2 did not impair accuracy of stimulus detection over the central visual field. The only alternative model with superior evidence than the null model was the one with Time as main effect $(\mathrm{BF}=1.98$, "anecdotal" evidence), indicating reduced accuracy at $\mathrm{T} 50$ relative to $\mathrm{T} 1$.

\section{RT}

The null model had stronger evidence ("anecdotal" to "substantial" evidence; $\mathrm{BF}=0.46$ to 0.16 ) than three of the models containing Stimulation (as main effect or interaction with Time). The "winning" model with the strongest evidence contained Time as a main effect ( $\mathrm{BF}=24.91$, "strong" evidence), suggesting learning effects for RT, marked by faster response at T50 relative to $\mathrm{T} 1$ in both stimulation conditions (Figure 4B). The other three alternative models containing Stimulation (as main effect or interaction with Time) had "substantial" to "strong" evidence relative the null $(\mathrm{BF}=4.42$ to 11.62$)$. However, each of these alternative models also contained Time as a main effect. To confirm that these models won over the null model because of the Time effect, we performed comparisons showing that Stimulation did not affect RT in addition to Time. We compared the model with Time as a main effect against each of these three alternative models by dividing the BF of the former by that of the latter. In all cases, results favored the Time model, offering "anecdotal" to "substantial" evidence (BF $=2.14$ to 5.63 ) supporting that Stimulation did not affect central vision RT in addition to Time. These results suggest that cTBS to V2 did not impact RT of detection over the central visual field immediately following or $50 \mathrm{~min}$ post-stimulation.

\section{Exploratory Analysis}

We explored whether cTBS differentially impacted subareas of the visual field by considering the factors of Hemifield (ipsilateral vs. contralateral) and Vision Type [foveal $\left(2^{\circ}\right)$ vs. parafoveal (4$\left.8^{\circ}\right)$ ]. Neither Hemifield nor Vision Type was found to have effects on accuracy and RT (see Supplementary Material).

\section{DISCUSSION}

The present study examined the effects of off-line, neuronavigated cTBS to $\mathrm{V} 2$ on central vision performance. 


\section{A Accuracy}

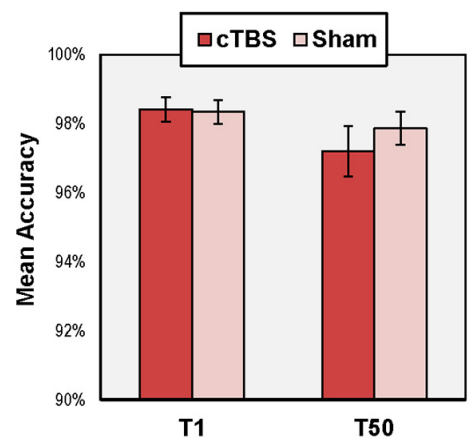

B RT

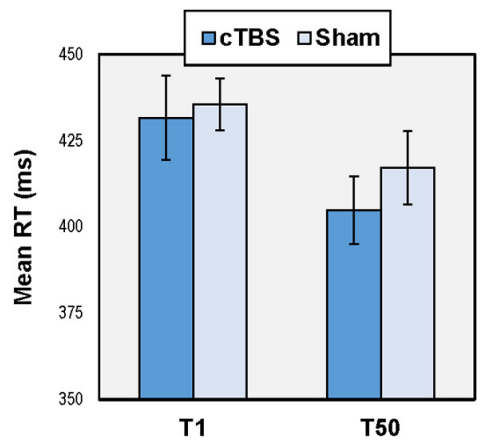

C

\section{Bayesian Model Comparison}

$B F=$ Evidence for Accuracy/RT (Alternative) Model / Evidence for ID-only (Null) Model

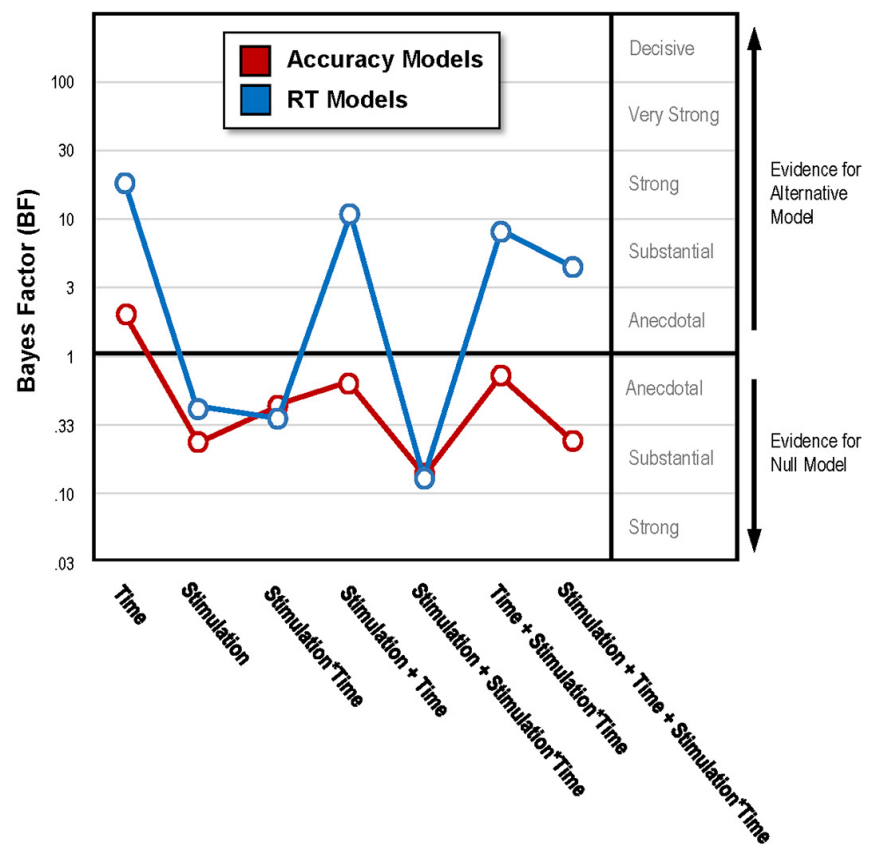

FIGURE 4 | Results of central vision performance analysis. (A) Mean accuracy for each condition and administration time; (B) Mean RT for each condition and administration time; (C) Bayesian model comparison results for all models relative to ID-only model. BF > 1 suggest change in vision performance while BF < 1 suggest no change. RT, reaction time; cTBS, continuous theta burst stimulation; V2, secondary visual cortex; ms, milliseconds; T1 and T50, 1- and 50-min post-stimulation.

Detection accuracy and RT over the central visual field were tested on a simple detection task following cTBS to V2 (at 1-min and 50-min post-stimulation) and compared to sham. We hypothesized that cTBS to V2 would not disrupt detection accuracy [based on results from lesion studies of the visual cortex (Merigan et al., 1993)] and would lead to slowed RT's [based on previous TMS results (Fiori et al., 2015)].

As expected, cTBS to V2 did not affect the accuracy of central vision performance on a simple detection task. This held when hemifield and vision type were accounted for (see Supplementary Material), which helped rule out the possibility that cTBS to V2 differentially affected detection over a particular portion of the visual field. This extends on previous negative findings [obtained using null hypothesis significance testing (Brückner and Kammer, 2014; Kaderali et al., 2015)] related to discrimination accuracy following visual cortex cTBS. Our findings conflicted with a previous report of low-level visual processing impairment following cTBS to early visual areas (Rahnev et al., 2013), but this was likely due to the methodological differences. The Rahnev et al. (2013) study, along with the majority of previous studies on this topic (Waterston and Pack, 2010; Clavagnier et al., 2013; Nuruki et al., 2013; Rahnev et al., 2013; Brückner and Kammer, 2014; Kaderali et al., 2015), used the phosphene threshold (PT) hotspot as their "early visual" target, whereas we used an anatomical map of V2 (Glasser et al., 2016).
Phosphene-based methods of localization can yield targets that vary widely between V1 through V3 (Schaeffner and Welchman, 2017). This can be problematic because, from lesion studies, we know that disruptions to different functional areas within "early occipital" regions have drastically different effects on visual processing [e.g., V1 lesions impair basic and complex visual processing, while V2 lesions impair complex processing only (Merigan et al., 1993)]. It is, therefore, possible that impairments reported in that study were caused by suppression of activity in V1 in some participants, which would be more likely to cause blind spots. In contrast, given our localization method, we were confident that V2 was stimulated in our study and thus our findings were specific to cTBS applied to V2 (see below for discussion of possible influences of intensity).

Contrary to hypotheses, cTBS to V2 did not impact RT of detection over the overall visual field nor specific subfields on a simple detection task. The discrepancy between this result and one previous study reporting slower RT following cTBS (Fiori et al., 2015) could be due to methodological differences. The task in the current study was relatively easy - a basic detection task with a fixed stimulus presentation duration (50 ms) - while Fiori et al. (2015) utilized staircase adaptation procedures to manipulate difficulty level, reducing stimuli viewing time in order to keep performance at $80 \%$ accuracy. The stimuli also varied in terms of complexity: we used coarsely discriminable targets 
while Fiori used more complex Gabor elements with varied orientations. Together, this placed more demands on participants in Fiori et al.'s (2015) study than those in the present study. This is important because, as discussed previously, lesions to V1 in macaques cause deficits on basic visual processing tasks, while lesions to V2 leave basic discrimination and contrast sensitivity unaffected (Merigan et al., 1993) but impair complex visual processing. Perhaps task demand/complexity is another reason why some have reported low-level visual impairments (Rahnev et al., 2013; Fiori et al., 2015) after early occipital cTBS, while our study and those using easier tasks reported no effects (Brückner and Kammer, 2014). Others have urged the importance of task complexity in TMS research (Waterston and Pack, 2010). Here we add that it is also important to carefully consider the specific visual area stimulated, because failure to consider both may have unforeseen interaction effects on vision performance.

Regardless of stimulation condition, time appeared to impact accuracy and RT, such that responses were less accurate but faster at T50 (relative to T1). It may be that participants were more comfortable with the task and thus were faster to respond (at a slight expense of accuracy).

Finally, one might question whether the stimulation intensity used in the present study was insufficient to adequately suppress V2 excitation. Although the intensity we used (80\% AMT) was lower than others (i.e., using resting motor threshold, phosphene threshold, or a fixed percentage of maximum stimulator output), several studies have successfully modulated behavior, perception, and cognition using visual cortex cTBS at intensities similar to ours (van Nuenen et al., 2012; Beck et al., 2015; Adam et al., 2016). This suggests that the absence of cTBS effects on simple visual detection performance in this study was not likely due to insufficient stimulation power. While visual impairments may be possible at much higher intensities of cTBS to V2, our goal was not to demonstrate this per se. Rather, we sought to assess whether blind spots would occur under a standard intensity (80\% AMT) (Rossi et al., 2009) most commonly adopted in cTBS research (Turi et al., 2021). In doing so, we demonstrated that cTBS delivered at standard intensities to V2 does not impair basic detection over the central visual field. However, this does not imply that cTBS to V2 at any intensities would leave vision intact. There is evidence that higher-intensity cTBS to early visual areas is capable of altering visual processing, but results are heterogeneous (Bertini et al., 2010; Waterston and Pack, 2010; Clavagnier et al., 2013; Chen et al., 2020).

These findings should be considered in light of several limitations. Our sample was modest and replications with larger sample sizes are needed. Additionally, the extant TMS literature (especially in the motor cortex) indicates that peak effects may not occur until $\sim 10-20$ min after stimulation (Huang et al., 2005). Therefore, the testing points used here might not have captured possible disruptions associated with peak effects. Future work should use additional time intervals between 0 - and 50 min following stimulation to assess for possible vision changes post-cTBS. This would be an important direction in future investigations to fully understand the time course of cTBS effects on central vision.
In summary, cTBS delivered at $80 \%$ AMT to V2 did not impair accuracy or RT of central vision detection during a simple detection task. Our findings provide further evidence consistent with previous reports that cTBS can be safely applied to V2 at standard intensities (80\% AMT) and does not disrupt basic early visual detection needed to perform tasks tapping higher-level visual processing or cognition. Replications with larger samples are needed to provide more definitive conclusions about the safety of cTBS in early visual cortices and effects on vision and behavior.

\section{DATA AVAILABILITY STATEMENT}

The raw data supporting the conclusions of this article will be made available by the authors, without undue reservation.

\section{ETHICS STATEMENT}

The studies involving human participants were reviewed and approved by the University of Michigan Medical School IRBMED. The patients/participants provided their written informed consent to participate in this study.

\section{AUTHOR CONTRIBUTIONS}

CL: methodology, software, investigation, data curation, formal analysis, visualization, and writing - original draft. ST: methodology, supervision, and writing - review and editing. TL: methodology, resources, supervision, and writing - review and editing. SR: methodology, software, and writing - review and editing. TG: software, formal analysis, visualization, and writing - review and editing. PG: investigation, data curation, visualization, and writing - review and editing. IT: conceptualization, methodology, funding acquisition, project administration, supervision, formal analysis, and writing review and editing. All authors contributed to the article and approved the submitted version.

\section{FUNDING}

This project was supported by a NARSAD Young Investigation Grant (to IT) and National Institute of Mental Health Grants (K23MH108823 and R01MH122491 to IT).

\section{ACKNOWLEDGMENTS}

Portions of these results were presented at the 31st Annual Albert J. Silverman Research Conference, University of Michigan.

\section{SUPPLEMENTARY MATERIAL}

The Supplementary Material for this article can be found online at: https://www.frontiersin.org/articles/10.3389/fnhum. 2021.709275/full\#supplementary-material 


\section{REFERENCES}

Abuleil, D., McCulloch, D., and Thompson, B. (2021). Visual Cortex CTBS Increases Mixed Percept Duration While A-TDCS Has No Effect on Binocular Rivalry. PLoS One 16:e0239349. doi: 10.1371/journal.pone.023 9349

Adam, J. J., Bovend'Eerdt, T. J., Schuhmann, T., and Sack, A. T. (2016). Allocentric Coding in Ventral and Dorsal Routes during Real-Time Reaching: evidence from Imaging-Guided Multi-Site Brain Stimulation. Behav. Brain Res. 300, 143-149. doi: 10.1016/j.bbr.2015.12.018

Allen, E. A., Pasley, B. N., Duong, T., and Freeman, R. D. (2007). Transcranial Magnetic Stimulation Elicits Coupled Neural and Hemodynamic Consequences. Science 317, 1918-1921. doi: 10.1126/science.114 6426

Barendregt, M., Dumoulin, S. O., and Rokers, B. (2014). Stereomotion Scotomas Occur after Binocular Combination. Vis. Res. 105, 92-99. doi: 10.1016/j.visres. 2014.09.008

Beck, B., Bertini, C., Haggard, P., and Làdavas, E. (2015). Dissociable Routes for Personal and Interpersonal Visual Enhancement of Touch. Cortex 73, 289-297. doi: 10.1016/j.cortex.2015.09.008

Bertini, C., Leo, F., Avenanti, A., and Làdavas, E. (2010). Independent Mechanisms for Ventriloquism and Multisensory Integration as Revealed by Theta-Burst Stimulation. Eur. J. Neurosci. 31, 1791-1799. doi: 10.1111/j.1460-9568.2010. 07200.x

Brainard, D. H. (1997). The Psychophysics Toolbox. Spat. Vis. 10, 433-436. doi: $10.1163 / 156856897 \times 00357$

Brückner, S., and Kammer, T. (2014). Is Theta Burst Stimulation Applied to Visual Cortex Able to Modulate Peripheral Visual Acuity? PLoS One 9:e99429. doi: 10.1371/journal.pone.0099429

Brückner, S., and Kammer, T. (2016). Modulation of Visual Cortex Excitability by Continuous Theta Burst Stimulation Depends on Coil Type. PLoS One 11:e0159743. doi: 10.1371/journal.pone.0159743

Cai, P., Chen, N., Zhou, T., Thompson, B., and Fang, F. (2014). Global versus Local: double Dissociation between MT+ and V3A in Motion Processing Revealed Using Continuous Theta Burst Transcranial Magnetic Stimulation. Exp. Brain Res. 232, 4035-4041. doi: 10.1007/s00221-014-4084-9

Chen, N., Chen, Z., and Fang, F. (2020). Functional Specialization in Human Dorsal Pathway for Stereoscopic Depth Processing. Exp. Brain Res. 238, $2581-$ 2588. doi: 10.1007/s00221-020-05918-4

Chiou, R., and Lambon Ralph, M. A. (2016). Task-Related Dynamic Division of Labor Between Anterior Temporal and Lateral Occipital Cortices in Representing Object Size. J. Neurosci. 36, 4662-4668. doi: 10.1523/jneurosci. 2829- 15.2016

Clavagnier, S., Thompson, B., and Hess, R. F. (2013). Long Lasting Effects of Daily Theta Burst RTMS Sessions in the Human Amblyopic Cortex. Brain Stimul. 6, 860-867. doi: 10.1016/j.brs.2013.04.002

Eldaief, M. C., Halko, M. A., Buckner, R. L., and Pascual-Leone, A. (2011). Transcranial Magnetic Stimulation Modulates the Brain's Intrinsic Activity in a Frequency-Dependent Manner. Proc. Natl. Acad. Sci. U. S. A. 108, 21229-21234. doi: 10.1073/pnas.1113103109

Fiori, F., Candidi, M., Acciarino, A., David, N., and Aglioti, S. M. (2015). The Right Temporoparietal Junction Plays a Causal Role in Maintaining the Internal Representation of Verticality. J. Neurophysiol. 114, 2983-2990. doi: 10.1152/jn. 00289.2015

First, M. B., Spitzer, R. L., Gibbon, M., and Williams, J. B. W. (2002). Structured Clinical Interview for DSM-IV-TR Axis I Disorders, Research Version, Patient Edition (SCID-I/P). New York: Biometrics Research.

Franca, M., Koch, G., Mochizuki, H., Huang, Y. Z., and Rothwell, J. C. (2006). Effects of Theta Burst Stimulation Protocols on Phosphene Threshold. Clin. Neurophysiol. 117, 1808-1813. doi: 10.1016/j.clinph.2006. 03.019

Glasser, M. F., Coalson, T. S., Robinson, E. C., Hacker, C. D., Harwell, J., Yacoub, E., et al. (2016). A Multi-Modal Parcellation of Human Cerebral Cortex. Nature 536, 171-178. doi: 10.1038/nature18933

Huang, Y. Z., Edwards, M. J., Rounis, E., Bhatia, K. P., and Rothwell, J. C. (2005). Theta Burst Stimulation of the Human Motor Cortex. Neuron 45, 201-206. doi: 10.1016/j.neuron.2004.12.033
Huang, Y. Z., and Rothwell, J. C. (2004). The Effect of Short-Duration Bursts of High-Frequency, Low-Intensity Transcranial Magnetic Stimulation on the Human Motor Cortex. Clin. Neurophysiol. 115, 1069-1075. doi: 10.1016/j. clinph.2003.12.026

Ilmoniemi, R. J., Ruohonen, J., and Karhu, J. (1999). Transcranial Magnetic Stimulation-a New Tool for Functional Imaging of the Brain. Crit. Rev. Biomed. Eng. 27, 241-284.

Janssen, A. M., Oostendorp, T. F., and Stegeman, D. F. (2015). The Coil Orientation Dependency of the Electric Field Induced by TMS for M1 and Other Brain Areas. J. Neuroeng. Rehabil. 12:47. doi: 10.1186/s12984-0150036-2

Jarosz, A. F., and Wiley, J. (2014). What Are the Odds? A Practical Guide to Computing and Reporting Bayes Factors. J. Probl. Solving 7:2.

Kaderali, S., Kim, Y. J., Reynaud, A., and Mullen, K. T. (2015). The Role of Human Brain Area HMT+ in the Perception of Global Motion Investigated With Repetitive Transcranial Magnetic Stimulation (RTMS). Brain Stimul. 8, 200-207. doi: 10.1016/j.brs.2014.11.001

Kleiner, M., Brainard, D., and Pelli, D. (2007). What's New in Psychtoolbox-3? Perception 36:14. doi: 10.1177/03010066070360s101

Klomjai, W., Katz, R., and Lackmy-Vallée, A. (2015). Basic Principles of Transcranial Magnetic Stimulation (TMS) and Repetitive TMS (RTMS). Ann. Phys. Rehabil. Med. 58, 208-213. doi: 10.1016/j.rehab.2015. 05.005

McKeefry, D. J., Gouws, A., Burton, M. P., and Morland, A. B. (2009). The Noninvasive Dissection of the Human Visual Cortex: using FMRI and TMS to Study the Organization of the Visual Brain. Neuroscientist 15, 489-506. doi: $10.1177 / 1073858409334424$

Merigan, W. H., Nealey, T. A., and Maunsell, J. H. R. (1993). Visual Effects of Lesions of Cortical Area V2 in Macaques. J. Neurosci. 13, 3180-3191. doi: 10.1523/jneurosci.13-07-03180.1993

Morey, R. D., and Rouder, J. N. (2015). BayesFactor: Computation of Bayes Factors for Common Designs. R Package Version 0.9.12-12.

Nuruki, A., Oliver, R., Campana, G., Walsh, V., and Rothwell, J. C. (2013). Opposing Roles of Sensory and Parietal Cortices in Awareness in a Bistable Motion Illusion. Neuropsychologia 51, 2479-2484. doi: 10.1016/j. neuropsychologia.2013.09.026

Nyffeler, T., Wurtz, P., Lüscher, H. R., Hess, C. W., Senn, W., and Pflugshaupt, T. (2006). Repetitive TMS over the Human Oculomotor Cortex: comparison of $1-\mathrm{Hz}$ and Theta Burst Stimulation. Neurosci. Lett. 409, 57-60. doi: 10.1016/j. neulet.2006.09.011

Pelli, D. G. (1997). The VideoToolbox Software for Visual Psychophysics: transforming Numbers into Movies. Spat. Vis. 10, 437-442. doi: 10.1163/ 156856897x00366

Quintana, D. S., and Williams, D. R. (2018). Bayesian Alternatives for Common Null-Hypothesis Significance Tests in Psychiatry: a Non-Technical Guide Using JASP. BMC Psychiatry 18:178. doi: 10.1186/s12888-018-1761-4

Rahnev, D., Kok, P., Munneke, M., Bahdo, L., de Lange, F. P., and Lau, H. (2013). Continuous Theta Burst Transcranial Magnetic Stimulation Reduces Resting State Connectivity between Visual Areas. J. Neurophysiol. 110, 1811-1821. doi: 10.1152/jn.00209.2013

Raz, N., and Levin, N. (2014). Cortical and White Matter Mapping in the Visual System-More than Meets the Eye: on the Importance of Functional Imaging to Understand Visual System Pathologies. Front. Integr. Neurosci. 8:68. doi: 10.3389/fnint.2014.00068

Rossi, S., Hallett, M., Rossini, P. M., Pascual-Leone, A., and Safety of Tms Consensus Groupr. (2009). Safety, Ethical Considerations, and Application Guidelines for the Use of Transcranial Magnetic Stimulation in Clinical Practice and Research. Clin. Neurophysiol. 120, 2008-2039. doi: 10.1016/j.clinph.2009. 08.016

Schaeffner, L. F., and Welchman, A. E. (2017). Mapping the Visual Brain Areas Susceptible to Phosphene Induction through Brain Stimulation. Exp. Brain Res. 235, 205-217. doi: 10.1007/s00221-016-4784-4

Silvanto, J., Muggleton, N., and Walsh, V. (2008). State-Dependency in Brain Stimulation Studies of Perception and Cognition. Trends Cogn. Sci. 12, 447-454. doi: 10.1016/j.tics.2008.09.004

Thut, G., and Pascual-Leone, A. (2010). A Review of Combined TMS-EEG Studies to Characterize Lasting Effects of Repetitive TMS and Assess Their Usefulness in 
Cognitive and Clinical Neuroscience. Brain Topogr. 22, 219-232. doi: 10.1007/ s10548-009-0115-4

Turi, Z., Lenz, M., Paulus, W., Mittner, M., and Vlachos, A. (2021). Selecting Stimulation Intensity in Repetitive Transcranial Magnetic Stimulation Studies: a Systematic Review between 1991 and 2020. Eur. J. Neurosci. 53, 3404-3415. doi: $10.1111 /$ ejn. 15195

van Nuenen, B. F., Helmich, R. C., Buenen, N., van de Warrenburg, B. P., Bloem, B. R., and Toni, I. (2012). Compensatory Activity in the Extrastriate Body Area of Parkinson's Disease Patients. J. Neurosci. 32, 9546-9553. doi: 10.1523/ jneurosci.0335-12.2012

Waterston, M. L., and Pack, C. C. (2010). Improved Discrimination of Visual Stimuli Following Repetitive Transcranial Magnetic Stimulation. PLoS One 5:e10354. doi: 10.1371/journal.pone.0010354

White, N., Roddey, C., Shankaranarayanan, A., Han, E., Rettmann, D., Santos, J., et al. (2010). PROMO: real-Time Prospective Motion Correction in MRI Using Image-Based Tracking. Magn. Reson. Med. 63, 91-105. doi: 10.1002/mrm. 22176
Conflict of Interest: The authors declare that the research was conducted in the absence of any commercial or financial relationships that could be construed as a potential conflict of interest.

Publisher's Note: All claims expressed in this article are solely those of the authors and do not necessarily represent those of their affiliated organizations, or those of the publisher, the editors and the reviewers. Any product that may be evaluated in this article, or claim that may be made by its manufacturer, is not guaranteed or endorsed by the publisher.

Copyright (c) 2021 Lasagna, Taylor, Lee, Rutherford, Greathouse, Gu and Tso. This is an open-access article distributed under the terms of the Creative Commons Attribution License (CC BY). The use, distribution or reproduction in other forums is permitted, provided the original author(s) and the copyright owner(s) are credited and that the original publication in this journal is cited, in accordance with accepted academic practice. No use, distribution or reproduction is permitted which does not comply with these terms. 